\title{
Índice tecnológico de las unidades de producción de tomate en invernadero en Tlahuitoltepec, Oaxaca
}

\author{
Technological index of tomato greenhouse production in Tlahuitoltepec, Oaxaca
}

Jesús Hernández-Ruíz ${ }^{1 *}$, Edgar Espinosa-Trujillo ${ }^{1}$, Ana Isabel Míreles-Arriagaํㅜ, Jorge Eric Ruiz-Nieto ${ }^{1}$

\begin{abstract}
RESUMEN
En los últimos cuatro años, la producción de tomate en invernadero en la región Mixe de Oaxaca ha comenzado a tomar relevancia entre las actividades agrícolas. El objetivo del trabajo fue determinar el índice tecnológico de las unidades de producción de tomate para proponer una categorización. Para la colecta de datos se aplicó una entrevista semiestructurada a 20 productores considerando información socioeconómica y productiva. El análisis descriptivo y de percentiles de datos fue mediante el programa SPSS. La superficie total de invernadero en el área de estudio fue de $16,950 \mathrm{~m}^{2}$. En promedio, cada productor cuenta con $871 \mathrm{~m}^{2}$ y un rendimiento promedio de $2.97 \mathrm{~kg} / \mathrm{m}^{2}$. El nivel tecnológico promedio fue de 0.56 en una escala de 0 a 1 . Se identificaron tres estratos de productores donde existe una relación entre el nivel tecnológico, ingreso, rendimiento y tamaño del invernadero. El 45\% de los productores vende su tomate a una cooperativa; el resto vende pequeños volúmenes en los mercados locales y regionales.
\end{abstract}

PALABRAS CLAVE

agricultura protegida, índice tecnológico, región mixe

\section{ABSTRACT}

The greenhouse tomato production in the Mixe region of Oaxaca has begun to be important among the agricultural activities in the last four years, whereby the objective of this study was to determine the technological index of the production units of tomato to propose a categorization based on this factor. For the collection of data, a semi-structured interview was applied to collect socio-economic and productive data. Descriptive and percentile analysis of data was performed through the SPSS program. The total greenhouse area in the study area was $16,950 \mathrm{~m}^{2}$, on average each producer owns $871 \mathrm{~m}^{2}$, with an average yield of $2.97 \mathrm{~kg} / \mathrm{m}^{2}$. The average technological level was 0.56 in a scale of 0 to 1 . A three-producer stratum was identified with a relationship between technological level, income, yield and greenhouse size. $45 \%$ of producers sell their tomatoes to a cooperative, the rest sell small volumes in local and regional markets.

KEYWORDS

protected agriculture, index technology, region mixe

\footnotetext{
${ }^{1}$ División de Ciencias de la Vida, Universidad de Guanajuato. Guanajuato, México.

*Autor para correspondencia. Ex hacienda El Copal, km 9 carretera Irapuato-Silao. 36500 Irapuato, Guanajuato, México.

Correo electrónico: hernandez.jesus@ugto.mx
} 


\section{INTRODUCCIÓN}

El cultivo de tomate, Solanum lycopersicum L. (Solanaceae), bajo invernadero en el estado de Oaxaca muestra un crecimiento continuo, que ha pasado de cero hectáreas en 1990 a 420 en 2014 (OEIDRUS 2011; Martínez-Gutiérrez et al. 2014). El Servicio de Información Agroalimentaria y Pesquera (SIAP) reporta 761 ha bajo ese sistema de producción, de las cuales $62.2 \%$ se localizan en la región Valles Centrales, $15.8 \%$ en la Cañada, $13.9 \%$ en la Mixteca Baja, $6.8 \%$ en el Istmo de Tehuantepec y 1.3\% en la Sierra Juárez (SIAP, 2016).

La producción de tomate bajo invernadero en la región Mixe es una actividad de nueva inserción (Anónimo, 2010) y, de acuerdo con el Comité Estatal del Sistema Producto de Tomate de Oaxaca, se han identificado cinco niveles de desempeño: muy bajo, bajo, medio, alto y muy alto. Asimismo, en esta entidad se encuentran las unidades de producción bajo invernadero más compactas, mismas que presentan un rendimiento por debajo de la media nacional, cuyo principal problema es la organización de los productores (Cih-Dzul et al. 2011; Martínez-Gutiérrez et al. 2014).

La limitante para obtener un resultado socioeconómico favorable en las unidades de producción agrícola es multifactorial pues involucra características como: organización de los productores, tecnología, magnitud de la producción y comercialización (Fong 2005; Martínez-Gutiérrez et al. 2014). Ante esto, se plantea el análisis de estas variables para caracterizar y estratificar grupos en función de las capacidades de producción (Köbrich et al. 2003; Righi et al. 2009).

A través de la caracterización y estratificación de las unidades de producción se puede abordar la estructura de producción agropecuaria y encontrar alternativas para diseñar estrategias que mejoren el desempeño e ingreso de productores (Benítez-García et al. 2015). La clasificación debe considerar principalmente variables del nivel tecnológico, ya que éstas se basan en las decisiones que el productor toma sobre las actividades agropecuarias que realiza; además, muestran mayor variación que los factores socioculturales (Bidogeza et al. 2009; Lema y Brescia 2001).

Caracterizar el índice tecnológico, así como los aspectos económicos y sociales con los que cuentan las unidades de producción de tomate en invernadero, se considera la base para definir estrategias que contribuyan al logro de un mayor desempeño del sistema de producción.
El nivel tecnológico es el producto de la interacción de conocimientos y técnicas disponibles que considera las relaciones entre insumos y herramientas con las que cuenta el productor agropecuario (BurgeSmani y Wheelwright 2004). Por lo anterior, el objetivo del presente trabajo fue determinar el índice tecnológico de las unidades de producción de tomate de la localidad de Santa María Tlahuitoltepec, Oaxaca, México, para proponer una categorización de los productores con base en este factor.

\section{Materiales Y MÉtodos}

\section{Sitio geográfico del estudio}

El marco geográfico del estudio incluyó las localidades pertenecientes a la cabecera municipal de Santa María Tlahuitoltepec (cuadro 1), ubicadas en la región mixe al noreste del estado de Oaxaca. El municipio se localiza entre los paralelos $17^{\circ} 03^{\prime}$ y $17^{\circ} 10^{\prime}$ de latitud norte, en los meridianos $95^{\circ} 58^{\prime}$ y $96^{\circ} 09^{\prime}$ de longitud oeste, y entre 1000 y 3400 masm (INEGI, 2010).

\section{Ciclo de producción evaluado}

Durante 2016 se realizaron entrevistas con productores de tomate en invernadero de las localidades pertenecientes a la cabecera municipal de Santa María Tlahuitoltepec. Se aplicaron entrevistas semiestructuradas de tres apartados: el primero abordó las características de la unidad de producción de tomate bajo invernadero y su ingreso; el segundo se enfocó en el proceso de producción y la tecnología empleada con el que se estimó el índice tecnológico, y el tercero se concentró en la comercialización de la hortaliza. Cada entrevista se aplicó a 20 productores seleccionados con base en el número total de invernaderos activos (cuadro 1).

\section{Características registradas}

Las características recabadas en las unidades de producción fueron sociodemográficas, sistema de producción, nivel tecnológico, rendimiento, superficie sembrada e ingresos económicos (cuadro 2). El ingreso monetario de las unidades de producción incluyó las percepciones corrientes recibidas en dinero provenientes de trabajo asalariado, del trabajo independiente, los ingresos derivados de la posesión 
Cuadro 1. Número de invernaderos con producción de tomate en las localidades de la cabecera municipal de Santa María Tlahuitoltepec, Oaxaca.

\begin{tabular}{|c|c|c|c|}
\hline LOCALIDAD & NúMERo de INVERNADERos & INVERNADEROS ACTIVOS & SUPERFICIE DE INVERNADEROS ACTIVOS $\left(\mathrm{M}^{2}\right)$ \\
\hline Guadalupe & 2 & 2 & 2,000 \\
\hline Santa Ana & 8 & 3 & 4,460 \\
\hline Cedro & 6 & 4 & 4,500 \\
\hline Tejas & 2 & 1 & 1,000 \\
\hline Flores & 8 & 6 & 2,500 \\
\hline Magueyal & 2 & 1 & 500 \\
\hline Frijol & 2 & 1 & 1,000 \\
\hline Laguna & 2 & 1 & 500 \\
\hline Red & 2 & 1 & 500 \\
\hline Total & 34 & 20 & 16,960 \\
\hline
\end{tabular}

Cuadro 2. Descripción de las variables registradas en las unidades de producción de tomate en invernadero en Santa María Tlahuitoltepec, Oaxaca.

\begin{tabular}{ll}
\hline \multicolumn{1}{c}{ VARIABLES } & \multicolumn{1}{c}{ Descripción } \\
\hline $\begin{array}{l}\text { 1. Escolaridad (ESC) } \\
\text { 2. Sexo (SEX) }\end{array}$ & $\begin{array}{l}\text { Primaria, secundaria, bachillerato, profesional y sin estudios } \\
\text { Mujer, hombre }\end{array}$ \\
3. Edad (ED) & Años \\
4. Integrantes de la familia (IF) & Número de personas que integran la unidad de producción familiar \\
5. Tamaño del invernadero (DIM) & Superficie de invernadero ( ${ }^{2}$ ) \\
6. Variedades de tomate (VT) & Número de variedades establecidas \\
7. Rendimiento (REN) & Kg/m² \\
8. Índice tecnológico (ITEC) & Tipo de invernadero, equipo y asesoría técnica. \\
9. Precio (PRE) & Precio por kg (\$) \\
10. Ingreso total anual (ING) & Ingresos por venta de tomate, venta de otros productos agropecuarios, \\
11. Porcentaje de ingreso de venta por & apoyos gubernamentales, trabajo no agropecuario y remesas \\
tomate (PIT) & PIT=ingreso de venta por tomate/ING \\
12. Destino de la producción & Autoconsumo y venta en mercado: local, regional o nacional \\
\hline
\end{tabular}

de activos físicos y las transferencias recibidas de instituciones públicas o privadas, así como de otros hogares (INEGI, 2008).

Se calculó el índice tecnológico (IT) con base en tres componentes: prácticas agrícolas $(P A)$, tipo de invernadero $(T I)$, y equipo y asesoría técnica $(E A T)$, dado que estos factores se utilizan para la clasificación de invernaderos (Ortega-Martínez et al. 2014). Se asignaron valores de ponderación para cada componente $(C)$, de tal forma que el $I T$ se calculó de la siguiente manera:

$$
I T=(0.5)(P A)+(0.25)(T I)+(0.25)(E A T)
$$

A las $P A$ se les asignó el mayor valor de ponderación (0.5) mientras que a TI y EAT se les brindó 0.25. La asignación de los valores de ponderación se realizó de acuerdo con el estudio de Benítez-García et al. (2015), donde se priorizan las prácticas agrícolas, dado que éstas resultan de la interacción de conocimientos y técnicas que emplea el productor.

Por otra parte, cada componente estuvo estructurado por subcomponentes $(S C)$, a los cuales se les asignaron otros valores de ponderación, de la siguiente forma: 
a. PA se integró de cuatro actividades: riego y fertilización $\left(S C_{1}\right)$, manejo de plagas y enfermedades $\left(S C_{2}\right)$, método de propagación y siembra $\left(\mathrm{SC}_{3}\right)$, y las prácticas de limpieza $\left(\mathrm{SC}_{4}\right)$. A cada una de éstas se le asignó un ponderador de 0.25 . Las buenas prácticas agrícolas son indispensables en los sistemas de producción agrícola, sobre todo el control de las plagas y enfermedades (Ruiz et al. 2011). Por lo anterior, se le asignó un peso de 0.25. Así, el cálculo del $P A$ ocurrió de la siguiente forma.

$$
P A=\sum_{i=1}^{n=4}(0.25)\left(S C_{i}\right)
$$

b. TI incluyó aspectos como los materiales de cubierta $\left(S C_{5}\right)$, estructura, forma $\left(S C_{6}\right)$ y según el rango de temperaturas a mantener; cada uno con un valor de 0.25 . La producción en invernadero tiene mayores beneficios en relación con la producción en campo abierto, dado que se tiene impacto positivo en el rendimiento y el control de plagas y enfermedades (Cih-Dzul et al. 2011). A estos subcomponentes se les asignó un peso de ponderación de 0.25 , cuyo cálculo quedó como sigue:

$$
T I=\sum_{i=5}^{n=6}(0.25)\left(S C_{i}\right)
$$

c. EAT consideró la existencia del apoyo técnico especializado $(S C 7)$ y la cantidad de equipo con la que contaba el productor (SC8); a cada uno de estos componentes se les asignó un valor ponderador de 0.50 . A este factor se le dio el mayor peso en la ponderación, ya que el personal técnico está conformado por agentes de la innovación en los sistemas de producción de tomate, por lo que su presencia es preponderante (Perilla et al. 2011). Además, $P A$ y $T I$ son componentes detonados con la asistencia técnica. De esta forma, se calculó el valor de EAT como sigue:

$$
E A T=\sum_{i=7}^{n=8}(0.5)\left(S C_{i}\right)
$$

\section{Categorización de productores}

Se estratificó con base en el índice tecnológico y tamaño del invernadero. En ambos casos se construyeron tres estratos mediante la división del número de productores en terciles, para lo cual se consideraron cuatro factores: tamaño de invernadero, rendimiento $\left(\mathrm{kg} / \mathrm{m}^{2}\right)$, manejo agrícola y nivel tecnológico. Para definir el nivel tecnológico se utilizó el concepto propuesto por BurgeSmani y Wheelwright (2004), quienes enfatizan que la tecnología es el producto de la interacción de conocimientos y técnicas disponibles que considera las relaciones entre insumos y herramientas, con base en las decisiones que el productor toma sobre las actividades productivas que realiza.

\section{Análisis de datos}

Los datos se procesaron bajo un análisis descriptivo y de percentiles mediante el uso del paquete Statistical Package for the Social Sciences (SPSS). Se realizó un análisis de conglomerados cualitativo mediante el programa JMP SAS v.10 (Sall et al. 2012) considerando las características sociodemográficas de las unidades de producción y el índice tecnológico (prácticas agrícolas, tipo de invernadero y asesoría técnica).

\section{RESULTADOS Y DISCUSIÓN}

\section{Características de las unidades de producción}

Los productores de tomate bajo invernadero en Santa María Tlahuitoltepec, Oaxaca; se caracterizan como minifundistas, con superficie sembrada promedio de $871 \mathrm{~m}^{2}$, la cual se encuentra por debajo de la media estatal 2,000 $\mathrm{m}^{2}$ (Martínez-Gutiérrez et al. 2014). A nivel nacional se estima que en la región sur de México (Guerrero, Oaxaca y Chiapas) cada productor siembra de dos a tres ha, mientras que en el sureste (Tabasco, Campeche, Quintana Roo) siembra dos.

En estas regiones habita el $61 \%$ de la población rural; sin embargo, las menores aportaciones de valor a la agricultura nacional se ubican en el sur $(9.3 \%)$ y el sureste $(1.5 \%)$. Esta condición limita el financiamiento en relación con el tamaño del predio, restringe su acceso a la agricultura protegida tecnificada, por lo que no cuentan con tecnología, infraestructura, capacitación, organización del capital humano, y aumenta la vulnerabilidad de la estructura productiva en el ámbito social y económico. Asimismo, a pesar que las pérdidas económicas son de un monto menor, la proporción del daño causado por los fenómenos climatológicos en su escaso capital es sumamente alta (Moreno et al. 2011).

El rendimiento promedio de las unidades de producción es de $2.97 \mathrm{~kg} / \mathrm{m}^{2}$, menor que el promedio 
estatal de 9.8 (SIAP 2016). Por su parte, FIRA (2007) considera que, para obtener ganancias, se requiere al menos un rendimiento de $35 \mathrm{~kg} / \mathrm{m}^{2}$ con tecnología media mientras que, para mercado nacional con baja tecnología, se requieren $15 \mathrm{~kg} / \mathrm{m}^{2}$.

La densidad de siembra (plantas $/ \mathrm{m}^{2}$ ) registró promedio de 2.5 plantas. Estas densidades de siembra son las más bajas reportadas en el estado de Oaxaca, dado que Martínez-Gutiérrez et al. (2014) reportan densidad promedio de 3.5 plantas $/ \mathrm{m}^{2}$. El reflejo de estos bajos rendimientos y densidades de siembra en sistemas de horticultura protegida sugiere que el papel preponderante de las familias rurales es mantener o aumentar la diversidad de las actividades agropecuarias y generar un entorno seguro en la producción de alimentos, y no la obtención de altos rendimientos (Sthapit et al. 2008).

De los entrevistados, $52 \%$ tiene escolaridad de nivel básico y $48 \%$ no cuenta con escolaridad de dicho nivel. Ésta es una característica sociocultural en las explotaciones agrícolas en zonas rurales (Luna-Méndez et al. 2013). El número de integrantes por familia es de cinco miembros en promedio; en este sentido, se infiere que el tamaño de la unidad familiar determina la cantidad de fuerza de trabajo disponible para las actividades productivas del invernadero dado que Martínez-Gutiérrez et al. (2014) reportan que 62.6\% de la mano de obra para la producción de tomate en Valles Centrales de Oaxaca es distribuida entre los miembros de la unidad familiar.

\section{Características del invernadero}

El 90\% de los invernaderos son de modelo baticenital, con material de construcción de acero y cubierta de plástico; el resto $(10 \%)$ ha sido construido con el modelo tipo túnel. De acuerdo con la clasificación de invernaderos de Ortega-Martínez et al. (2014), este tipo de estructuras se ubica en la clase A: estructuras de invernaderos unitarios o en batería. El principal origen de los invernaderos fue el financiamiento gubernamental $(70 \%)$, seguido del financiamiento de una asociación civil (25\%) y, en menor grado, la gestión por parte un organismo descentralizado de la administración pública federal (5\%).

\section{Tipo de tomate}

Los años de experiencia en la producción de tomate dentro de la comunidad son de un rango máximo de nueve y mínimo de uno. El tipo de tomate que se cultiva principalmente, es saladette Cid (90\%); el resto de los productores cultiva el tomate tipo bola.

\section{Prácticas agronómicas}

Un elevado porcentaje (90\%) de productores realiza rotación y asociación de cultivos en sus invernaderos y aplica abonos orgánicos; el resto de los productores sólo se dedica a la producción de tomate. Las plántulas son adquiridas mayormente en empresas privadas: $50 \%$ en Agroservicios Mixe Semillas; 20\% en maquiladora Hortícola, y el resto de los productores $(30 \%)$ obtiene sus propias semillas mediante selección del fruto.

En cuanto a la utilización de fertilizantes, los productores utilizan siete marcas comerciales $\left(\right.$ Calcinit $^{\circledast}, \mathrm{NKS}^{\circledast}$, Micromix $^{\circledR}$, Sop ${ }^{\circledR}$, Triple 18, Ultrasol ${ }^{\circledR}$, Sulmag $\left.{ }^{\circledR}\right)$, de las cuales el mayor porcentaje $(80 \%)$ corresponde a Calcinit ${ }^{\circ}$. El volumen que utilizan es en promedio de 50 bultos, por cada ciclo de producción. Los agricultores durante un ciclo de producción de tomate mencionaron que la plaga con mayor persistencia es la mosca blanca [Bemisia tabaci (Gennadius), Hemiptera: Aleyrodidae]. Por lo anterior, $80 \%$ asperja al cultivo para combatir a esa plaga, 15\% para los áfidos [Myzus persicae (Sulzer) y Aphis gossypii (Glover), Hemiptera: Aphididae] y 5\% para el minador (Liriomyza sativae Blanchard, Diptera: Agromyzidae). La asesoría técnica que perciben los productores es por parte de una asociación civil (40\%) y empresas privadas de la región (50\%); el 10\% no percibe asesoría técnica.

\section{Ingreso por ventas de tomate}

La inversión en promedio por unidad de producción durante un ciclo es de 18,150 pesos, de los cuales invierten $60 \%$ en fertilizantes e insecticidas, 35\% en plántulas, $5 \%$ en herramientas; la mano de obra utilizada es familiar.

El precio en el mercado local de 8.00 a 12.00 pesos/ $\mathrm{kg}$. En el mercado regional la venta se realiza en taras de $20 \mathrm{~kg}$, donde el precio promedio es de 120.00 pesos. En la población se encuentra activa una Sociedad de Productores Rural denomina Xaam Ko'on (tomate tlahuitoltepecano), a la que se encuentran afiliados $45 \%$ de los productores. Esta organización ha permitido a los productores tener un comprador seguro, mientras que el resto de los productores no tiene forma de realizar ventas consolidadas de su producción. La venta del tomate producido en la 


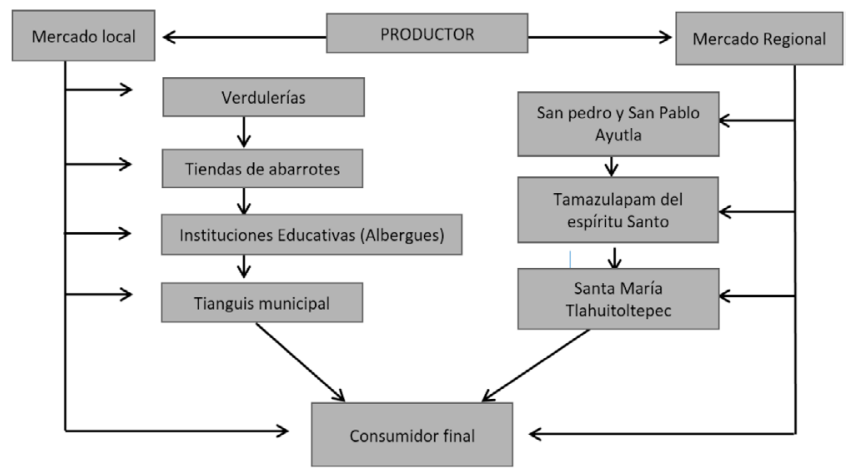

Figura 1. Canales de comercialización del tomate en Santa María Tlahuitoltepec, Oaxaca, México.

comunidad de Tlahuitoltepec se realiza en el mercado regional (60\%) y en el mercado local (40\%) (figura 1$)$.

\section{Índice tecnológico}

El índice tecnológico promedio de los 20 productores es de 0.56 y sólo dos productores tienen el nivel más alto 0.70 (figura 2). Esto indica que la tecnología para el cultivo de tomate bajo invernadero en Tlahuitoltepec, Oaxaca, es relativamente bajo. En este sentido el Comité Estatal Sistema Producto de Tomate de Oaxaca ha identificado cinco niveles de desempeño: muy bajo, bajo, medio, alto y muy alto, donde cerca de $72 \%$ de las unidades de producción tienen un desempeño por debajo de la media nacional (Martínez-Gutiérrez et al. 2014).

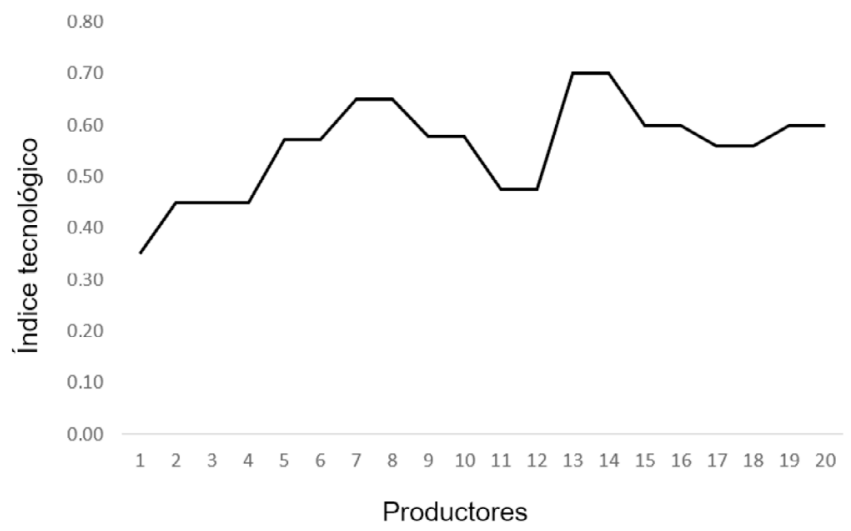

Figura 2. Índice tecnológico de productores de tomate en invernadero en Santa María Tlahuitoltepec, Oaxaca, México.

La categorización por cuantiles con base en el índice tecnológico (IT) muestra que $25 \%$ de los productores que conforman el estrato uno $(Q 1)$ cuenta con valor de IT menor a 0.46 , destina el $59 \%$ de la producción de tomate para venta, posee el menor valor de superficie de invernadero $\left(375 \mathrm{~m}^{2}\right)$ y rendimiento $(2.475 \mathrm{~kg} /$ $\mathrm{m}^{2}$ ); además, la producción de tomate sólo significa $20 \%$ de sus ingresos familiares, los cuales son de $1,687.00$ pesos. En las familias que dependen menos de la producción de tomate, sus ingresos provienen de actividades fuera de la actividad hortícola, donde predomina el ingreso por remesas del extranjero. Este fenómeno es común en las zonas rurales de Oaxaca, en donde han existido altas tasas de emigración (Barron y Rello, 2000).

El estrato dos $(Q 2)$ lo integran productores que poseen un $I T$ de 0.47 a 0.59 . Ellos destinan $80 \%$ de la producción de tomate para venta, poseen una superficie de invernadero de $937 \mathrm{~m}^{2}$, rendimiento de $2.475 \mathrm{~kg} / \mathrm{m}^{2}$, y la producción de tomate significa $40 \%$ de sus ingresos familiares (5,325.00 pesos). El tercer estrato $(Q 3)$ está integrado por los productores que cuentan con IT mayor a 0.60 , quienes destinan $86 \%$ de la producción de tomate para venta, poseen el mayor valor de superficie de invernadero $\left(10,204 \mathrm{~m}^{2}\right)$ y rendimiento $\left(3.12 \mathrm{~kg} / \mathrm{m}^{2}\right)$, y la producción de tomate significa $75 \%$ de sus ingresos familiares $(10,204.00$ pesos) (cuadro 3).

En este sentido Cih-Dzul et al. (2011) y García et al. (2015) mencionan que el grado de tecnología de un productor se manifiesta en las variedades que cultiva, el número de plantas por metro cuadrado, control de malezas, fertilización y control de plagas. Todas estas actividades aplicadas conjuntamente pueden aumentar su ingreso y mejorar su capacidad productiva.

La categorización por cuantiles en base a la superficie muestra que $25 \%$ de los productores que conforman el estrato uno $(Q 1)$ cuenta con la menor superficie de invernadero $\left(<750 \mathrm{~m}^{2}\right)$ e integrantes promedio por familia (tres). La edad promedio del encargado de la unidad de producción es 58 años y el valor de índice tecnológico es de 0.52. Quienes conforman el grupo dos (Q2) poseen una superficie de invernadero de 751 a 1,000 $\mathrm{m}^{2}$, los integrantes promedio por familia son cinco y la edad promedio del encargado de la unidad de producción es 50 años; el valor de índice tecnológico es de 0.58 . Los del grupo tres $(Q 3)$ cuentan con la mayor superficie de invernadero $\left(>1,001 \mathrm{~m}^{2}\right)$ e integrantes promedio por familia (seis); la edad promedio del encargado de la unidad de producción es 45 años y el valor de índice tecnológico es de 0.59 (cuadro 4).

Considerando la clasificación por superficie de invernaderos, se infiere que el tamaño de invernadero con el que disponen las unidades de producción de tomate bajo invernadero está ligado directamente al 
Cuadro 3. Categorización de productores con base en su nivel tecnológico.

\begin{tabular}{|c|c|c|c|c|c|c|}
\hline $\begin{array}{l}\text { Estrato } \\
\text { Cunantil }\end{array}$ & $\begin{array}{c}\text { ÍNDICE } \\
\text { TECNOLÓGICO }\end{array}$ & $\begin{array}{c}\text { INGRESO } \\
\text { (\$) }\end{array}$ & $\begin{array}{l}\text { SUPERFICIE } \\
\qquad\left(\mathbf{M}^{2}\right)\end{array}$ & $\begin{array}{c}\text { RendimiEnto } \\
\mathbf{K G}^{\prime} / \mathbf{M}^{2}\end{array}$ & $\begin{array}{c}\text { VENTA DE } \\
\text { TOMATE } \\
\text { (\%) }\end{array}$ & $\begin{array}{c}\text { INGRESO } \\
\text { VENTA DE } \\
\text { TOMATE } \\
\%(\%)\end{array}$ \\
\hline Q1 (25\%) & $<0.46$ & 1687 & 375 & 2.47 & 59 & 20 \\
\hline Q2 (50\%) & $0.47-0.59$ & 5325 & 937 & 2.67 & 80 & 40 \\
\hline Q3 (75\%) & $>0.60$ & 10204 & 1052 & 3.12 & 86 & 75 \\
\hline
\end{tabular}

Cuadro 4. Categorización de los productores por superficie.

\begin{tabular}{|c|c|c|c|c|c|c|c|}
\hline $\begin{array}{l}\text { Estrato } \\
\text { CuAntil }\end{array}$ & $\begin{array}{c}\text { SUPERFICIE } \\
\left(\mathbf{M}^{2}\right)\end{array}$ & $\begin{array}{c}\text { INGRESO } \\
\text { (\$) }\end{array}$ & $\begin{array}{c}\text { ÍNDICE } \\
\text { TECNOLÓGICO }\end{array}$ & $\begin{array}{c}\text { RENDIMIENTO } \\
\text { KG/M }^{2}\end{array}$ & INTEGRANTES & $\begin{array}{c}\text { Venta } \\
\text { (\%) }\end{array}$ & EDAD \\
\hline Q1 & $<750$ & 2,298 & 0.52 & 2.71 & 3 & 67 & 58 \\
\hline Q2 & $751-1000$ & 4,700 & 0.58 & 2.76 & 5 & 86 & 50 \\
\hline Q3 & $>1,001$ & 1,1472 & 0.59 & 3.00 & 6 & 86 & 45 \\
\hline
\end{tabular}

tamaño de familia, lo cual les facilita destinar más mano de obra a la producción y mejorar el rendimiento, efecto observado por Martínez-Gutiérrez et al. (2014), quienes mencionan que el trabajo al interior de cada unidad de producción (menor a 2,000 $\mathrm{m}^{2}$ ) en el estado de Oaxaca es principalmente familiar. En contraparte, unidades de producción con superficies superiores a los 2,000 $\mathrm{m}^{2}$ presentan limitantes productivas, como: incertidumbre fitosanitaria, costos de operación para controlar temperatura y humedad relativa y la escala de producción (Moreno et al. 2011).

El análisis de conglomerados por el método de Ward mostró que, a una distancia de 5.23, se conforman tres grupos, los cuales coinciden con la categorización definida por los percentiles de índice tecnológico (figura 3).

\section{Conclusiones}

Las unidades de producción de tomate bajo invernadero de Santa María Tlahuitoltepec, en promedio tienen niveles bajos de superficie $\left(871 \mathrm{~m}^{2}\right)$ y rendimiento $\left(2.97 \mathrm{~kg} / \mathrm{m}^{2}\right)$. El trabajo organizativo al interior es principalmente familiar, lo que limita el acceso a incentivos para gestionar capacitación e inversión financiera, dado que la gestión organizativa $\mathrm{y}$ de apoyos gubernamentales enfocados a la agricultura protegida requieren la creación de figuras legalmente constituidas. La categorización con base en el índice tecnológico permitió observar tres estratos

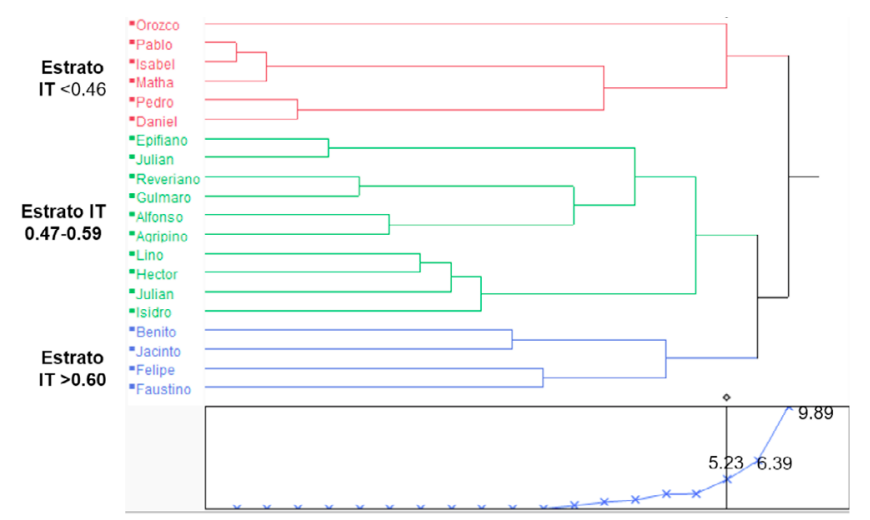

Figura 3. Análisis de conglomerados de productores en invernadero en el municipio de Santa María Tlahuitoltepec, Oaxaca, México. de unidades de producción, donde existe una relación entre el nivel tecnológico, ingreso, rendimiento y el tamaño del invernadero.

El primer grupo lo conforman productores que en promedio tienen índice tecnológico $<0.46$, poseen $<375$ $\mathrm{m}^{2}$ de invernadero, tienen rendimientos de $2.47 \mathrm{~kg} / \mathrm{m}^{2}$, la producción de tomate significa $20 \%$ de sus ingresos familiares y la unidad familiar está conformada en promedio por tres integrantes.

Los productores del segundo grupo poseen en promedio un índice tecnológico de 0.47 a 0.59 , cuentan 
con $937 \mathrm{~m}^{2}$ de invernadero, tienen rendimientos de $2.67 \mathrm{~kg} / \mathrm{m}^{2}$, la producción de tomate significa $40 \%$ de sus ingresos familiares y la unidad familiar está conformada por cinco integrantes.

El tercer grupo cuenta con productores que en promedio tienen un índice tecnológico de $>0.60$, poseen $1,052 \mathrm{~m}^{2}$ de invernadero, rendimientos de $3.12 \mathrm{~kg} / \mathrm{m}^{2}$, la producción de tomate significa $75 \%$ de sus ingresos familiares y la unidad familiar está conformada en promedio por seis integrantes.

A partir de estos resultados, será posible proponer y desarrollar estrategias que conlleven a un mejor manejo, así como a la implementación de técnicas agrícolas para optimizar el uso de los recursos en función de las necesidades de cada estrato de productores, y de manera general implementar talleres de capacitación enfocados al manejo agronómico que permita mejorar los rendimientos. 


\section{LiTERATURA CITADA}

Anónimo [internet]. 2010. Plan de desarrollo municipal Santa María Tlahuitoltepec [cited 2016 Oct 18]. Disponible en: https: //finanzasoaxaca.gob.mx/pdf/inversion_ publica /pmds/08_10/437.pdf

Barron MA, Rello F. 2000. The impact of the tomato agroindustry on the rural poor in Mexico. Agricultural Economics 23(3): 289-297. https://doi.org/10.1016/S01695150(00)00090-6

Benítez-García E, Jaramillo-Villanueva JL, Escobedo-Garrido S, Mora-Flores S. 2015. Caracterización de la producción y del comercio de café en el Municipio de Cuetzalan, Puebla. Agricultura, Sociedad y Desarrollo 12(2): 181-198.

Bidogeza JP, Berentsen PBM, De Graaff J, Oude Lansink AGJM. 2009. A typology of farm households for the Umutura Province in Rwanda. Food Security 1(3): 321335. https:// doi.org/10.1007/s12571-009-0029-8

BurgeSmani RA, Wheelwright SC. 2004. Strategic management of technology and innovation. Reading 1(1): 66-77.

Cih-Dzul IR, Jaramillo-Villanueva JL, Tornero-Campante MA, Schwentesius-Rindermann R. 2011. Caracterización de los sistemas de producción de tomate (Lycopersicum esculentum Mill.) en el Estado de Jalisco, México. Tropical and Subtropical Agroecosystems 14(2): 501512.

[FIRA] Fideicomisos Instituidos en Relación con la Agricultura [internet]. 2007. Agricultura protegida: cultivo de tomate en invernadero. Costos de cultivo y análisis de rentabilidad 2006 [cited 2016 Ene 04]. Disponible en: http://www.fira.gob.mx/Nd/TOMATE\%20INVERNADERO $\% 201 \% 20$ Norte $\% 20-\% 20$ Analisis $\% 20$ de $\% 20$ Costos.pdf

Fong C. 2005. La teoría de los recursos y capacidades: fundamentos microeconómicos. Universidad de Guadalajara. Guadalajara, México.

García EI, Aguilar J, Bernal R. 2015. La agricultura protegida en Tlaxcala, Méjico: La adopción de innovaciones y el nivel de equipamiento como factores para su categorización. Teuken Bidikay 2: 193-212.

[INEGI] Instituto Nacional de Estadística y Geografía e Informática [internet]. 2008. Encuesta Nacional de Ingresos y Gastos de los Hogares (ENIGH) [cited 2016 Nov 12]. Disponible en: http://www.inegi.org.mx/est/contenidos/proyectos/encuestas/hogares/enogh

[INEGI] Instituto Nacional de Estadística y Geografía e Informática. 2010. Localidades de la República Mexicana, 2010, escala: 1:1. Obtenido de Principales resultados por localidad (ITER). Censo de Población y Vivienda 2010. Comisión Nacional para el Conocimiento y Uso de la Biodiversidad (CONABIO). México, D. F.

Köbrich C, Rehman T, Khan M. 2003. Typification of farming systems for constructing representative farm models: two illustrations of the application of multi-variate analyses in Chile and Pakistan. Agricultural Systems 76(1): 141-157. https://doi.org/10.1016/S0308521X(02)00013-6
Lema D, Brescia V. 2001. Medición del cambio tecnológico, la productividad y la eficiencia del sector agropecuario. Taller Internacional "La Modelización en el Sector Agropecuario". Universidad de Buenos Aires. Buenos Aires, Argentina.

Luna-Méndez N, Jaramillo-Villanueva JL, Ramírez-Juárez J, Escobedo-Garrido S, Bustamante-González Á, Campos-Ríos G. 2013. Tipología de unidades de producción de nuez de castilla en sistema de producción tradicional. Agricultura, Sociedad y Desarrollo 10(3): 283-303.

Martínez-Gutiérrez GA, Díaz-Pichardo R, Juárez-Luis G, Ortiz-Hernández YD, López-Cruz JY. 2014. Caracterización de las unidades de producción de tomate en invernaderos de Oaxaca. Agricultura, Sociedad y Desarrollo 11: 153-165.

Moreno A, Aguilar J, Luévano A. 2011. Características de la agricultura protegida y su entorno en México. Revista Mexicana de Agronegocios 29: 763-774.

[OEIDRUS] Oficina Estatal de Información para el Desarrollo Rural Sustentable [internet]. 2011. Invernaderos Datos Básicos 2008 [cited 2016 Oct 18]. Disponible en: http://www.oeidrus-oaxaca.gob.mx/invernaderos2008. htm

Ortega-Martínez LD, Ocampo-Mendoza J, Sandoval-Castro E, Martínez-Valenzuela C, Huerta-De La Peña A, Jaramillo-Villanueva JL. 2014. Caracterización y funcionalidad de invernaderos en Chignahuapan Puebla, México. Revista Bio Ciencias 2(4): 261-270. https:// doi. org/10.15741/revbio.02.04.04

Perilla A, Rodríguez LF, Bermúdez LT. 2011. Estudio técnico-económico del sistema de producción de tomate bajo invernadero en Guateque, Sutatenza y Tenza (Boyacá). Revista Colombiana de Ciencias Hortícolas 5(2): 220-232.

Righi E, Pacini GC, Dogliotti S, Aguerre V, Rossing WAH. 2009. Farm typology identification by multi-variate analysis as a method to scale-up results of integrated impact assessment. AgSAP Conference 2009. Egmond aan Zee, The Netherlands.

Ruiz RE, Ruiz JA, Guzmán S, Pérez EdeJ. 2011. Manejo y control de plagas del cultivo de tomate en Cintalapa, Chiapas, México. Revista Internacional de Contaminación Ambiental 27(2): 129-137.

Sall J, Lehman A, Stephens M. 2012. JMP start statistics: a guide to statistics and data analysis using JMP. SAS Institute.576 pp.

[SIAP] Servicio de Información Agroalimentaria y Pesquera [internet]. 2016. Datos preliminares de la producción de tomate 2016 [cited 2016 Oct 18]. Disponible en: http://infosiap.siap.gob.mx:8080/agricola_siap_gobmx/ ResumenProducto.do

Sthapit B, Rana R, Eyzaguirre P, Jarvis D. 2008. The value of plant genetic diversity to resource-poor farmers in $\mathrm{Ne}$ pal and Vietnam. International Journal of Agricultural Sustainability 6(2): 148-166. 